\title{
Photostructural Changes and Electrical Switching in Amorphous Chalcogenides: Bond Waves in Thin Films
}

\author{
Elena A. Chechetkina
}

Institute of General and Inorganic Chemistry of Russian Academy of Sciences (1980-2011), Moscow, Russia

Correspondence to: Elena A. Chechetkina, eche2010@yandex.ru

Keywords: Glassy Semiconductors, Self-Organization, Photostructural Changes, Electrical Switching

Received: January 2, $2018 \quad$ Accepted: February 25, $2018 \quad$ Published: February 28, 2018

Copyright (C) 2018 by authors and Scientific Research Publishing Inc.

This work is licensed under the Creative Commons Attribution International License (CC BY 4.0).

http://creativecommons.org/licenses/by/4.0/

\section{c) (i) Open Access}

\section{ABSTRACT}

Photostructural changes and electrical switching are the well-known features of amorphous chalcogenides, also known as glassy semiconductors. Although the both phenomena were intensively studied experimentally and have a wide practical application, their nature is debated up to now. I propose a new approach that considers glass as a self-organizing system owing to characteristic instability of chemical bonding in the form of bond wave. The bond wave model is shown to be suitable for explanation of the observed effects in thin films under the action of light or electrical field, a result that opens a new way for understanding and managing the processes in glassy semiconductors.

\section{INTRODUCTION}

Amorphous chalcogenides represent a relatively new class of materials, glassy semiconductors, whose discovery of sixty years ago [1] has created a puzzle of how can semiconductor exist in the non-crystalline state [2]. During the following intensive study, which was initiated in the Kolomiets' laboratory [3, 4], many principal features of these materials were soon established, electrical switching (ES) and photostructural changes (PSC) being the most popular from both experimental and practical points of view [5].

Despite of intensive experimental study for a more than half-century, the nature of PSC and ES remains under discussion. Here I propose not a next model but a principally new approach, which is based on understating glass as a self-organizing system [6]. To be more concrete, the bond wave model is used for explanation of the known experiments and for planning of the new ones, emphasizing the usage of information fields to control underlying processes.

\section{SELF-ORGANIZATION AND THE BOND WAVE MODEL}

\subsection{The Bond Wave Model}

This model stands on two supports: the theory of chemical bonding and the theory of self-organization. These two aspects are considered independently in glass community up to now. Chemical bonding is a 
very popular topic, beginning from the continuous random network after Zachariasen [7]; CRN represents an ideal glass structure composed from "normal" covalent bonds (CB), the same that exist in a related crystal. Self-organization is a fresher term in glass theory; it was introduced and developed by Boolchand et al. $[8,9]$ to denominate the so-called "intermediate phase" realizing in a definite composition range of complex (two- and more component) glass-forming systems; it is attributed to the stress-free state of CRN considered in frames of the so-called "topological theory" of glass structure [10-12]. It should be noted, however, that such "self-organization" seems rather self-assembling [13] than classical self-organization introduced by Haken $[14,15]$ and Prigogine $[16,17]$ for understanding the processes of evolution and adaptation in non-equilibrium complex systems.

Classical self-organizing system is an ordered formation that interacts with its surrounding by means of dissipative patterns characteristic for this system - therefore the first name is "self". A concrete state of such a system depends not only on the conditions (temperature, pressure, etc.) but also on the path, by which the system went to the point of observation. In this sense, self-organizing system possesses "memory", a property that is reflected in glass theory as the "landscape" picture or the "fictive temperature" parameter. Nevertheless, self-organization in its classical sense has not been applied to the glassy state so far, excluding the author's bond wave approach. This approach is based on the chemical bonding instability (when one and the same atoms can be linked by one or another bond of different parameters) and the collective character of this instability in the form of bond wave, which means the spatiotemporal correlation between elementary acts of the chemical bonds exchange. Bond waves are considered as the dissipative patterns characteristic for glass-forming substances. In Figure 1, the first image of the bond wave, which was proposed in 1987 [18], is shown.

Bond wave means the appearance of sub-lattice of the $\Lambda=\lambda_{\mathrm{D}}$ period in common CRN. One can ask what is the need for alternative bonds and corresponding sub-lattice? The need for special "soft" bands/cuts containing some "weakened bonds", which divide the stressed regions of CRN, was recognized

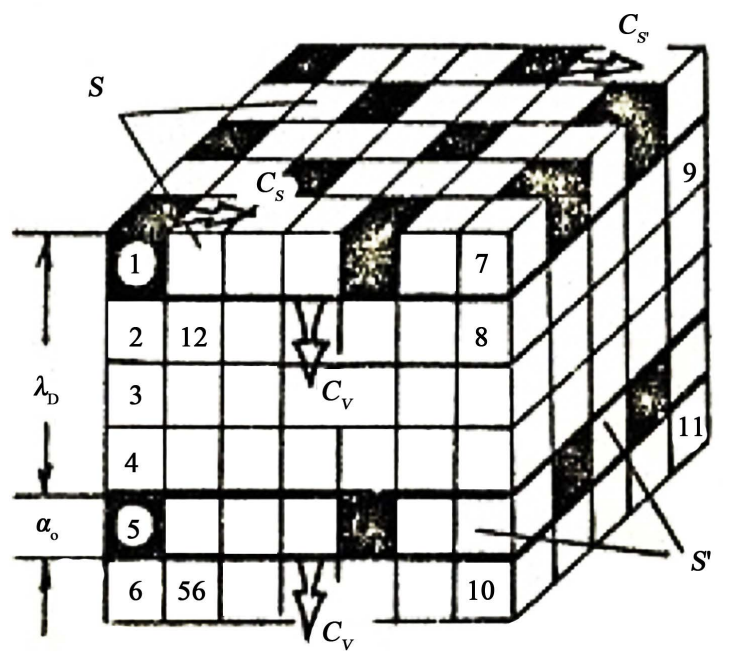

Figure 1. Element of bond wave with two wavefronts (S) populated with alternative bonds (black cubes) together with "normal" covalent bonds (white cubes) after [18]. Here $\lambda_{\mathrm{d}}$. is the wavelength; $\mathrm{C}_{\mathrm{V}}$ and $\mathrm{C}_{\mathrm{S}}$ are the velocities of $3 D$ and $2 D$ bond waves; $a_{0}$ is the average elementary length of the bond exchange cell. 
more that half-century ago $[19,20]$. Some later Ovshinsky has forwarded the concept of bond option as a necessary condition for realization of the non-crystalline state [21]. The most consistent and detailed investigation of the chemical bonding problem in the context of glassy state was made by Dembovsky, who went from hypothetical "quasimolecular defects" in 1981 [22] to hypervalent bonds (HVB), the latter being investigated intensively in typical inorganic glass formers by means of quantum-chemical method. Some examples are given in Figure 2 (see [26] for a full review).

Unfortunately, $\mathrm{HVB}$ was not found in $\mathrm{SiO}_{2}$ [27], and the $\mathrm{HVB}$ in $\mathrm{GeO}_{2}$, which is composed of 5- and 6-fold coordinated silicon atoms $\left(\mathrm{Si}_{5}\right.$ and $\left.\mathrm{Si}_{6}\right)$ and 3-fold coordinated oxygen $\left(\mathrm{O}_{3}\right)$ atom, was observed only when the calculation cluster was pressed by shifting of its boundaries [28]. Interestingly, $\mathrm{Si}_{6}$ was found in the case of complex $\mathrm{SiO}_{2}-\mathrm{P}_{2} \mathrm{O}_{5}-\mathrm{Na}_{2} \mathrm{O}$ system [29]; and, as concerns pure silica, there were found non-hypervalent "soft" 3c-4e (three-center, four-electron) bonds [30]. Therefore, hypervalency seems to be not so important property for glass formation as an ability for bond alternation itself, therefore, I use the term alternative bond (AB) for general description of self-organization in non-crystalline network.

As to the wavy-like sub-lattice in CRN (Figure 1), one can see it both directly-by fracture of brittle glass, which denudes the frozen wavefronts [31], and indirectly-by analyzing glass features, namely, the reduced melting/boiling temperatures [32], the first sharp diffraction peak (FSDP) [33, 34], and temperature dependence of viscosity [35]. Moreover, the basic problems of glass transition, secondary relaxation, and hierarchy of order in glass can be naturally treated from the bond wave point of view [36]. To be more specific, the three types of order, including long-range order of the $\Lambda$ period, are shown in Figure 3.

\subsection{Bond Waves in Thin Films}

The previous application of the bond wave model for interpretation of glass features [31-36] have concerned macroscopic properties, in which the 3D (volume) effects due to the $\Lambda$-lattice prevail. In the present case of films, the coexistence of $2 \mathrm{D}$ and $3 \mathrm{D}$ effects becomes essential. In addition, the two types of surfaces, a "free" surface (opposite to the substrate) and the "boundary" surface between substance and substrate, should be considered.

The state of bond wave at the free surface of a sample is unclear now. One can suppose the ripple-like surface waves like those observed on the river surface when wind blows. Really, the ripples of the $\Lambda \approx 700$ $\mathrm{nm}$ period were observed in the Se-S glass after the action of femtosecond laser [37]. The problem is that self-organized laser-induced ripples exist in non-glass forming substances too, for example in polycrystalline $\mathrm{Ti}[38,39]$; the phenomena itself was have been discovered as far as in 1965 [40]. Therefore, let us consider how can the bond waves characteristic for glass-forming substances interact with the film substrate. The possible effects are shown schematically in Figure 4.

One should remember that film is prepared in "hot" conditions, when the initial temperature is much higher than the glass transition temperature; in any case the incoming particle energy is very high. Therefore, the growing films can produce dissipative patterns in the form of $3 \mathrm{D}$ bond waves, which interact with
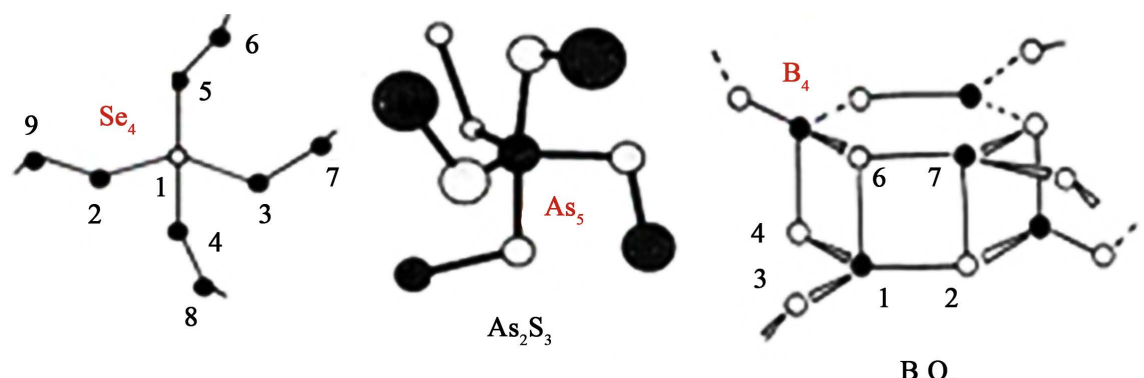

Figure 2. Metastable hypervalent configurations in Se [23] $\mathrm{As}_{2} \mathrm{~S}_{3}$ [24] and $\mathrm{B}_{2} \mathrm{O}_{3}$ [25] obtained by $a b$ initio quantum-chemical modeling in the cluster approach. 


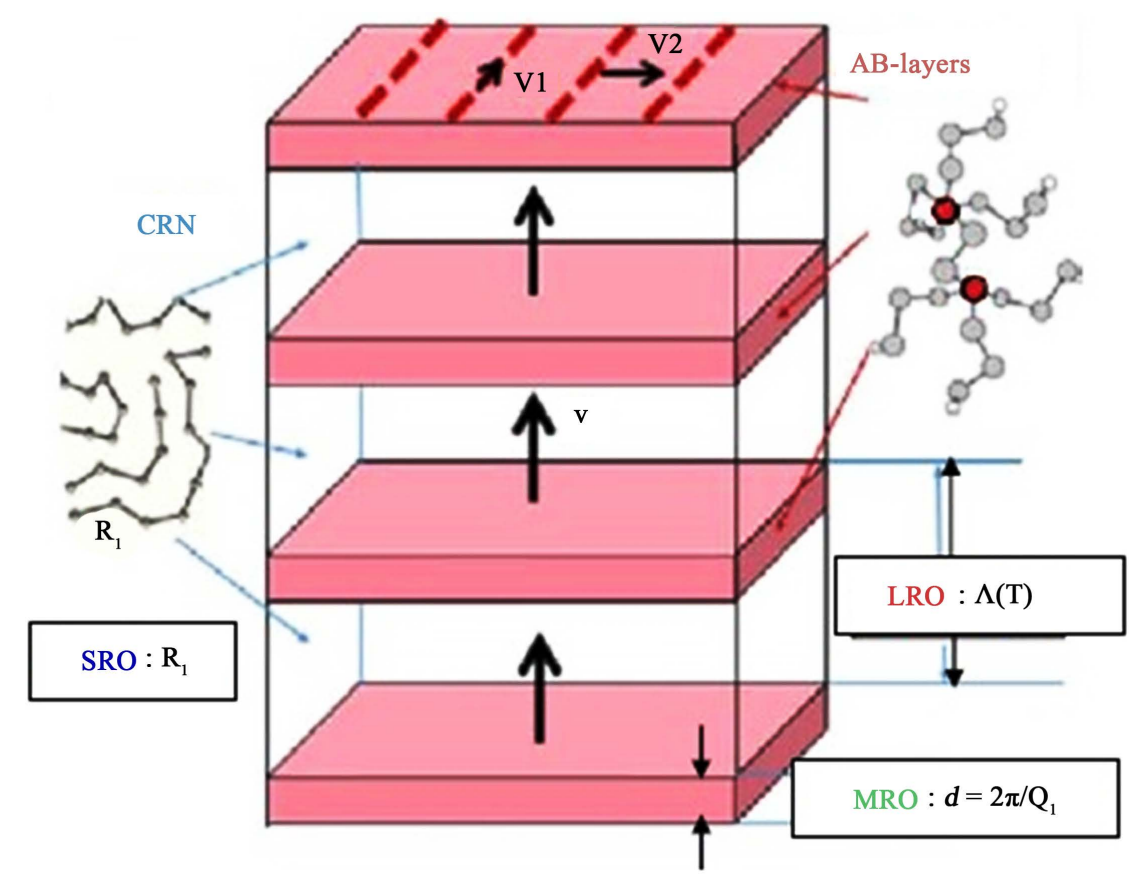

Figure 3. Scheme of the bond wave structure and dynamics after [36]. SRO is the short-range order of the $R_{1}$ size (the CB length is obtained from diffraction experiment treated as radial diffraction function), MRO is the medium-range order of the wavefront thickness of the $d$ size (corresponds to the directly observed FSDP reflex at the $\mathrm{Q}_{1}$ scattering vector), $\mathrm{LRO}$ is the long-range order of the $\Lambda$ period. Velocities $V, V_{1}$ and $V_{2}$ correspond to the $3 D, 2 D$ and $1 \mathrm{D}$ bond waves respectively; the $3 \mathrm{D}$ bond wave is refrozen $(\mathrm{V}>0)$ above $T_{g}$, the glass transition temperature. AB-layers represents the $3 \mathrm{D}$ bond wave wavefronts.

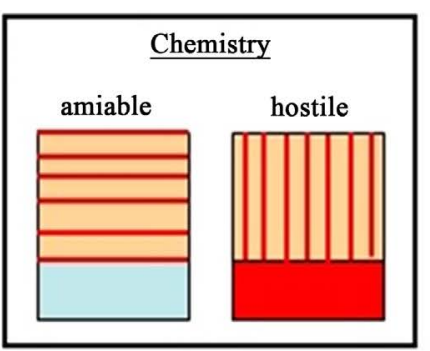

(a)

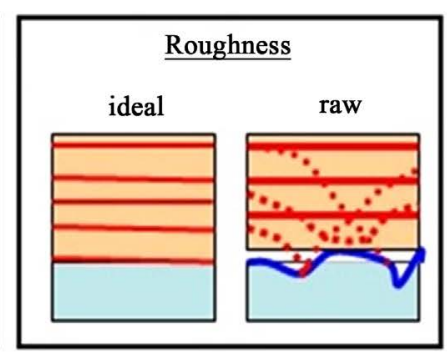

(b)

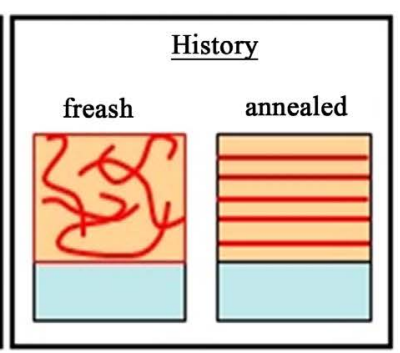

(c)

Figure 4. Influence of substrate nature (a), the substrate roughness (b), and the film history (c), on the internal sub-structure of a film.

a relatively cold substrate and then frozen on it at the end of the process. Interactions of two types seems to be essential. First is the "chemical" interaction since substrate composition can be amiable, neutral or hostile for the wavefront of the forming bond wave, so the wave orients in a conforming direction (Figure 4(a)). Second is the "physical" interaction due to the substrate roughness (Figure 4(b)): raw boundary can reflex incoming bond waves in a random way, so creating a complex sub-structure, which then freezes in a 
solid film.

The well-known fact is that properties of fresh (as-prepared) and annealed films can differ significantly. It is also known that the angle of evaporating influences on the following film behavior [41]. Such facts concerning film history become quite clear when considering film as the self-organizing system owing to formation of bond waves during its preparation. For example, when film is prepared in extreme conditions, such as flash evaporation onto the cooled surface, the film body has no a time to generate bond wave before solidifying-only the fragments of wavefronts are frozen in the solid film (Figure 4(c), on the left). The subsequent annealing at $T>T_{g}$, however, can not only refreeze these fragments but also organize them into $3 \mathrm{D}$ bond wave, which then frozen with the formation of solid film, whose ordered structure is shown in Figure 4(c), on the right.

\subsection{Bond Waves and Information Fields}

A mutual feature of both photostructural changes (PSC) and electrical switching (ES) is their development under the action of external energy sources, namely, laser irradiation for PSC and electric field for ES. Note that classical self-organizing systems realizes only in the streams of energy, matter (not necessarily) and information. The latter stream in the form of information field [15] gives the direction in which such a system evolves; in our case the direction in which bond wave propagates. The streams of information and energy can have the same nature, e.g., thermal source ensures both temperature-dependent formation of alternative bonds with their collective movement through CRN and the bond wave direction by temperature gradient, grad $T$. For example, let us consider the fracture of glassy rod from the point of view that fracture denudes the wavefronts of bond wave(s) that had developed before solidification.

There are two features observed in Figure 5. First is the coexistence of two families of denuded surfaces: one arranged alone the rod axis and other in the perpendicular direction. The families correspond to the two bond waves which intersect each other without distortion. Such a solitonic behavior of bond waves was emphasized by me earlier [31,34]. Second feature is the shape of fracture that can clear the action of information fields existing in the course of the rod drawing. Longitudinal wavefronts are directed by the pressure gradient, which forces the AB-layers (blue family in the insertion) to orient along grad $\boldsymbol{P}$. Although grad $T$ is of the same direction, the temperature gradient orients bond wave in the opposite way (see red family in the insertion). The "red" wavefronts should move to the heater, if we consider them as an ordered and so relatively "cold" formations-or from the heater, if they should dissipate the income of thermal energy. The result will the same: in the both cases bond wave moves along the grad $T$ direction, so corresponding wavefronts are disposed in the perpendicular position.

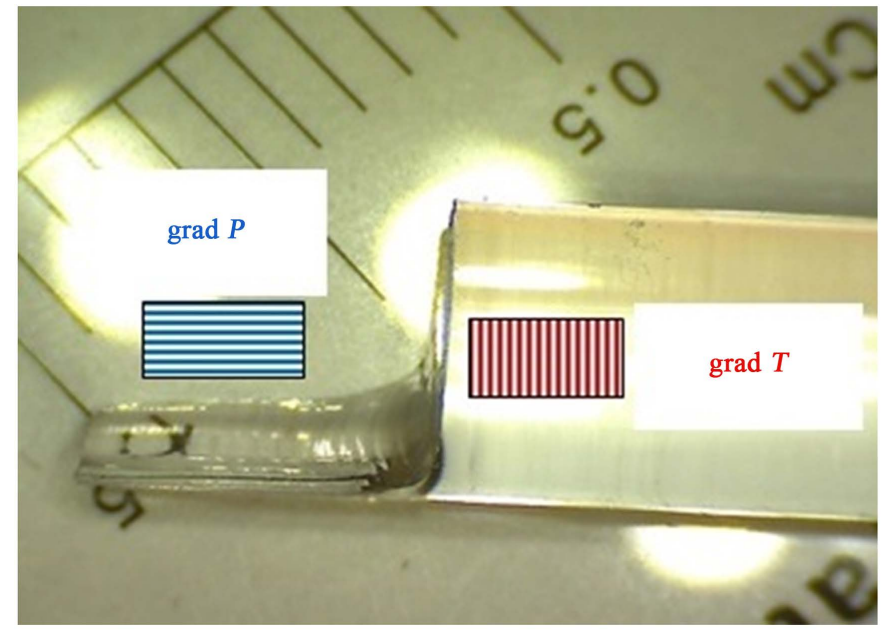

Figure 5. Typical fracture of glassy road with two families of the frozen wavefronts. 
Note that the energy stream not necessary be the information stream at the same time, and vice versa. We have observed just the information action of weak magnetic field on viscosity of softening $\left(T>T_{g}\right)$ selenium glass $[42,43]$. The field is "weak" in the sense that the elementary energy of magnetic field was incommensurably low relatively to that of thermal energy ( $\mu_{B} H \ll k T$ for $H \approx 250$ Oe and $T \approx 300 \mathrm{~K}$ ), so thermal motion should destroy all the field induced effects. Nevertheless, a giant change of viscosity was indicated: up to 6 times in alternating magnetic field, when changing the field direction at resonance conditions (50 Hz field frequency versus $310 \mathrm{~K}$ sample temperature) [43].

\section{APPLICATION OF THE BOND WAVE MODEL}

\subsection{Photostructural Changes (PSC)}

PSC are so rich and diverse that it is impossible to consider them in one way from phenomenological point of view. A concrete effect depends on the film composition, thickness and history; on the illumination conditions (energy, intensity, time, polarization, etc.); other factors being also important (substrate, impurities, atmosphere, etc.). As a result, one can observe both classical "darkening", i.e., the red-shift of the optical absorption edge, and "bleaching" (blue-shit), both expansion and construction of the illuminated region, as well as various anisotropic effects (see ref. [44] for introducing review). Therefore, I propose not a unified model for PSC but a unified bond wave approach by which a specific understanding of each concrete case can be achieved, and the following experiments can be planned.

Let us consider the so-called giant photo-expansion as an example. The phenomenon of a relatively large (5\%) expansion of the $\mathrm{As}_{2} \mathrm{~S}_{3}$ film irradiated by the sub-gap $(2.0 \mathrm{eV})$ laser with the formation of smooth "lens" on the irradiated surface region was discovered 1994 [45]. Later Tanaka et al. [46] have proposed a general "geometric" picture and a "microscopic" model to explain this phenomenon. I agree with the picture, when the sub-gap light can excite a relatively deep cylinder, which then is pulled out from the film body-but not with the model that considers elementary processes in the region of about $1-10$ $\mathrm{nm}$. To my opinion, to create an object of a regular form and a relatively large dimension (the lens diameter is $\sim 1-10 \mu \mathrm{m})$ a collective feedback between the excited microscopic regions should exist.

To understand the phenomena let us apply the bond wave model, as it is shown in Figure 6. Since the effect is observed only for the annealed films, let us take the initial state "1" from Figure 4. At the beginning of illumination (state " 2 ") additional alternative bonds appear in the excited region and, when decreasing the distance between them, these alternative bonds are organized into mobile/refrozen bond wave (red lines are the section of moving wavefronts) which spreads in the $h v$ direction (state " 3 "). Note that irradiation plays a complex role of both energy stream and information stream. Since alternative bonds demand additional space as compared with "rigid" covalent bonds, the excited cylinder tends to expand, however, the rigid surrounding of the frozen bond wave (blue lines) resists this expansion. As a result, a mobile system of refrozen wavefronts is pulling out, thus forming a growing "lens" on the film surfacestate " 4 ". After cessation of illumination, the ejected bond wave freezes (from red to blue), thus forming a solid lens on the film surface-state " 5 ". Note that not only the lens but also the cylindric region under it

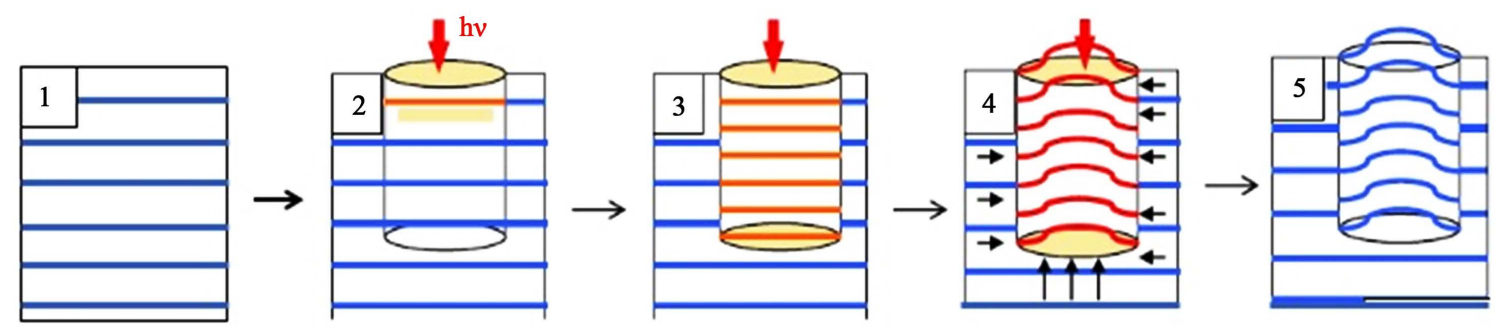

Figure 6. Scheme of the lens formation under film illumination. Blue lines and curves are the section of wavefronts belonging to the frozen bond wave(s), red lines and curves are those for the excited bond wave. 
has another sub-structure than surrounding, thus, this is not only the form effect-the region below the lens should have different properties (optical, etc.) than the residual film body.

Now let us consider the deviations from this picture. It is known that lenses not always appear under laser illumination of the film surface. For example, the $\mathrm{Ga}_{10} \mathrm{Ge}_{2} \mathrm{~S}_{65}$ film was also expanded after illumination, but its surface looked like a peeling skin: no lenses and even no ripples were observed there [47]. This is a result of an extra high light energy employed: the photons of $h v \gg E_{g}$ cannot penetrate the film, and even if alternative bonds are generated on the surface, they cannot organize even $2 \mathrm{D}$ bond wave, only a randomly damaged and thus expanded region is formed.

In the other work [48], when the band gap illumination $\left(h v \approx E_{g}\right)$ was applied to the $\mathrm{GeS}_{2}-\mathrm{Ga}_{2} \mathrm{O}_{3}$ film, no lens or pillow appear, instead, a pulled cylinder was observed. The applied light also cannot create a deep excited well, however, it acts more gently than in the previous case of the super-gap irradiation. Therefore, the newly generated alternative bonds are firstly organized into $2 \mathrm{D}$ bond waves on the film surface. For further self-organization into 3D bond wave there needs a space for its spreading-and the excited region provide it by means of extraction of the material from the inferior layer. The cylinder grows during illumination in the direction of the energy source. After cessation of illumination the "extracted" cylinder freezes because of the freezing of 3D bond wave that was formed it.

Interestingly, various reliefs (lenses, cylinders, needles, hollows, etc.) can be obtained also by electron beam [49], thus, the nature of the energy source is not very essential for formation of the self-organizing patterns in glassy films.

\subsection{Electrical Switching (ES)}

ES is the phenomenon of a sharp increase of conductivity of non-crystalline chalcogenide film when approaching the critical value of the electric field intensity, the so-called threshold voltage, $\mathrm{V}_{\mathrm{th}}$. The following negative-resistance state can be either reversible, when at the coming down side of income impulse the film returns to its initial high-resistant state, or transforms into a new low-resistance state, which means formation of a crystalline channel between the electrodes. The first case is known as the threshold switching (TES), and the latter is known as the memory switching (MES). The latter is of a great practical importance because of its usage in the digital technology for writing, storage and recording of information, a fact that is probably leads to recognition Ovshinsky, who was the designer of first memory cells, the father of electrical switching phenomenon. Nevertheless, one should not forget that the discovery of MES in 1968 [50] was published of 5 years later the TES discovery in 1963 by Russian scientists Kolomiets \& Lebedev [51].

Both TES and MES have the negative-resistance state, after which the following return (TES) or crystallization (MES) finish the cycle. The end depends on the crystallization ability of a substance. For example, in the Ge-Se-Bi system it is possible to obtain both TES and MES in different ranges of Bi concentration, ( $\mathrm{Bi}$ is known to be the crystallization agent) [52]. In frames of the bond wave model one can propose the following scheme of the ES process.

The initial film state is considered as the frozen bond waves; in Figure 7(a) the section of two bond waves, and so two families of wavefronts are shown by green dotted lines. Because of the electrical field applied (lower and upper electrodes, \pm ) the jumps of bonding electrons accompanying bond exchange (covalent bond into alternative bond, and back) begins. Since the jumps are forced in the electric field direction, reorientation of alternative bonds $(\mathrm{AB})$ proceeds in such a way that the family of inclined wavefronts (remember that wavefront is the place of $\mathrm{AB}$ concentration) disappears. As a result, the only one bond wave, whose wavefronts are disposed in the electric field direction remains. Because of the increased concentration of alternative bonds in the wavefronts of this merged bond wave the jumps in the strings disposed along the field direction becomes more intense (Figure 7(b)). Increasing voltage creates new $A B$ along the strings and so increasing current density. At the threshold voltage $V_{\text {th }}$ concentration of $A B$ in a string becomes so large that the one-dimensional bond wave (1D BW) begins, ensuring the enhanced mechanism of current flow (Figure 7(c)). Bond waves, being dissipative patterns, transforms energy of electric field into the thermal energy, the process of Joule heating created new strings, which increase total 


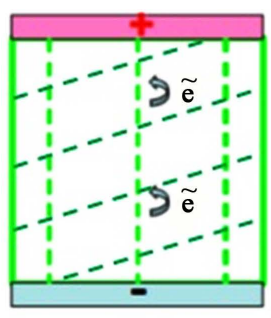

(a)

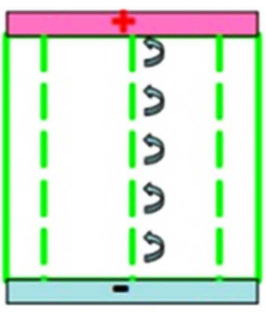

(b)

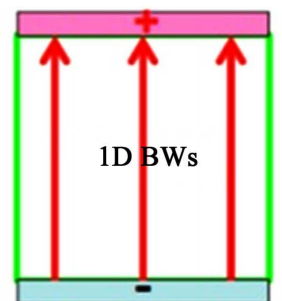

(c)

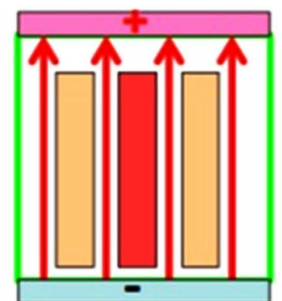

(d)

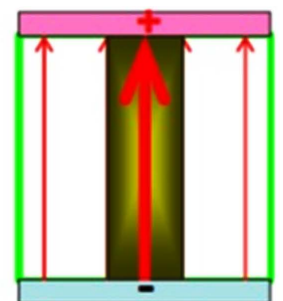

(e)

Figure 7. The stages of electrical switching: electron jumps along the primary strings of alternative bonds (a), field-induced reorientation and generation of additional $A B(b)$, formation of $1 \mathrm{D}$ bond waves with the "bonding" current along the strings (c), generation of additional strings due to Joule heating (d), formation of a crystalline channel in case of the memory switching (e).

current, and so on; this is the positive-feedback process that provides the negative-resistance state (Figure $7(d))$.

Then two ways open. If the heating is strong and/or crystallization ability of a substance is high, the mostly active strings create the superheated region which then crystallizes with the formation of a low-resistant channel (Figure 7(e))-this is MES. Otherwise the coming down income impulse returns the film into the state " $b$ ", and the process repeats under the action of the following impulses-this is the way of TES. Of course, concrete experiments bring new phenomenological details and natural laws (e.g., the $\mathrm{V}_{\text {th }}$ dependence on chemical composition of the film [53], on its thickness [54] and temperature [55], which can be used to improve the model and/or introduce other aspects into consideration. Once more way to manage the process was proposed by us more than thirty years ago [56]. It was shown that weak magnetic field, whose energy is incommensurably smaller than $k T$, influences on the switching TES parameters in the Ge-Se-Bi system; for example, the effect on the $\left(\mathrm{GeSe}_{3.5}\right)_{90} \mathrm{Bi}_{10}$ sample reaches a maximum at about $500 \mathrm{Oe}$, [8\% increase of $\mathrm{V}_{\text {th }}$ and $6 \%$ decrease of $\mathrm{V}_{\text {th }}$ for the $\left(\mathrm{GeSe}_{3.5}\right)_{90} \mathrm{Bi}_{10}$ sample], and then remains constant, at least, up to 2500 Oe. The field weakness together with quick saturation of the effect points to the self-organization nature of the process of electrical switching.

\section{CONCLUSION}

A unified approach for understanding photostructural changes and electrical switching in glassy semiconductors is proposed. This approach is based on the notions about self-organization and the model of bond wave, the latter being spatiotemporal correlation between elementary acts of bond exchange with participation of alternative bonding states. This approach/model gives opportunity to explain the peculiarities of the both phenomena and opens a new way for an effective management of underlaying processes by means of information fields.

\section{REFERENCES}

1. Goryunova, N.A. and Kolomiets, B.T. (1956) New Glassy Semiconductors. Izv. AN SSSR (in Russ.), 20, 1496-1501.

2. Mott, N.F. and Davis, E.A. (1979) Electron Processes in Non-Crystalline Materials. Clarendon Press, Oxford.

3. Kolomiets, B.T. (1964) Vitreous Semiconductors (I, II). Physica Status Solidi, 7, 359-372, 713-731. https://doi.org/10.1002/pssb.19640070302

4. Kolomiets, B.T. (1976) Electrical and Optical Properties of Vitreous Chalcogenide Semiconductor Films. Thin Solid Films, 34, 1-7. https://doi.org/10.1016/0040-6090(76)90114-0 
5. Adam, J.-L. and Chang, X. (2014) Chalcogenide Glasses. Woodhead Publ., Elsevier.

6. Zhang, W. (2015) Selforganizology: The Science of Self-Organization. World Sci. Publ., Singapore, Hackensack, London.

7. Zachariasen, W.H. (1932) The Atomic Arrangement in Glass. Journal of the American Chemical Society, 54, 3841-3851. https://doi.org/10.1021/ja01349a006

8. Boolchand, P., Georgiev, D.G. and Goodman, B. (2001) Discovery of the Intermediate Phase in Chalcogenide Glasses. Journal of Optoelectronics and Advanced Materials, 3, 703-720.

9. Boolchand, P., Lucovsky, G., Phillips, J.C. and Thorpe, M.F. (2005) Self-Organization and Physics of Glassy Networks. Philosophical Magazine, 85, 3823-3838. https://doi.org/10.1080/14786430500256425

10. Phillips, J.C. (1979) Topology of Covalent Non-Crystalline Solids. I. Short-Range Order in Chalcogenide Alloys. Journal of Non-Crystalline Solids, 34, 153-181. https://doi.org/10.1016/0022-3093(79)90033-4

11. Thorpe, M.F. (1983) Continuous Deformation in Random Networks. Journal of Non-Crystalline Solids, 57, 355-370. https://doi.org/10.1016/0022-3093(83)90424-6

12. Mauro, J.C. (2011) Topological Constraint Theory of Glass. American Ceramic Society Bulletin, 90, 31-37.

13. Yan, L.-T. (2017) Self-Assembling Systems: Theory and Simulation. Wiley-VCH, Weinheim.

14. Haken, H. (1978) Synergetics. An Introduction. Non-Equilibrium Phase Transition and Self-Organization in Physics, Chemistry and Biology. Springer, Berlin-Heidelberg, New York.

15. Haken, H. (2006) Information and Self-Organization: A Macroscopic Approach to Complex Systems. Springer, Berlin-Heidelberg, New York.

16. Nicolis, G. and Prigogine, I. (1977) Self-Organization in Nonequilibrium Systems. Wiley, Weinheim.

17. Prigogine, I. (1980) From Being to Becoming: Science and Complexity in the Physical Sciences. Freeman, New-York.

18. Chechetkina, E.A. (1987) One-Dimension and Two-Dimension Continua in Glasses. In: New Ideas in Glass Physics, Mendeleev's Institute of Chemical Technology, Moscow, 149-156.

19. Tilton, L.W. (1957) Noncrystal Ionic Model for Silica Glass. Journal of Research of the National Bureau of Standards, 59, 139-154. https://doi.org/10.6028/jres.059.015

20. Robinson, H.A. (1965) On the Structure of Vitreous $\mathrm{SiO}_{2}$. I. A New Pentagonal Dodecahedral Model. Journal of Physics and Chemistry of Solids, 26, 209-222. https://doi.org/10.1016/0022-3697(65)90148-4

21. Ovshinsky, S.R. (1985) Basic Anticrystalline Chemical Bonding Configurations and Their Structure and Physical Properties. Journal of Non-Crystalline Solids, 75, 161-168. https://doi.org/10.1016/0022-3093(85)90217-0

22. Dembovsky, S.A. (1981) Connection of Quasidefects with Glass Formation in the Substances with High Lone-Pair Electron Concentration. Materials Research Bulletin, 16, 1331-1338.

https://doi.org/10.1016/0025-5408(81)90105-7

23. Zyubin, A.S., Grigoriev, F.V. and Dembovskii, S.A. (2001) Quantum-Chemical Modeling of Hypervalent Defects in Amorphous Selenium. Russian Journal of Inorganic Chemistry, 46, 1350-1357.

24. Zyubin, A.S. and Dembovskii, S.A. (2004) Quantum-Chemical Modeling of Deformation and Bond Defects in Vitreous $\mathrm{As}_{2} \mathrm{~S}_{3}$. Russian Journal of Inorganic Chemistry, 49, 734-741.

25. Zyubin, A.S., Dembovsky, S.A. and Kondakova, O.A. (1998) Quantum-Chemical Study of the Network Modification in Vitreous $\mathrm{B}_{2} \mathrm{O}_{3}$. Journal of Non-Crystalline Solids, 224, 291-298.

https://doi.org/10.1016/S0022-3093(97)00474-2

26. Dembovsky, S.A. and Chechetkina, E.A. (2001) Glassy Materials Clarified through the Eyes of Hypervalent Bonding Configurations. Journal of Optoelectronics and Advanced Materials, 3, 3-18. 
27. Zyubin, A.S. and Dembovskii, S.A. (1996) Quantum-Chemical Search of "Weak Points" in Vitreous $\mathrm{SiO}_{2}$. Russian Journal of Inorganic Chemistry, 41, 657-662.

28. Zyubin, A.S., Kondakova, O.A. and Dembovskii, S.A. (1997) Quantum Chemical Simulation of the Transformation of Continuous Random Network in the Vitreous $\mathrm{GeO}_{2}$. Glass Physics and Chemistry, 23, 58-66.

29. Miyabe, D., Takahashi, M., Tokuda, Y. and Ushiono, T. (2005) Structure and Formation Mechanism of Six-Fold Coordinated Silicon in Phosphosilicate Glasses. Physical Review B, 71, Article ID: 172202. https://doi.org/10.1103/PhysRevB.71.172202

30. Dianov, E.M., Sokolov, V.O. and Sulimov, V.B. (1997) Calculation of Soft Atomic Potentials Caused by Three-Center Bonds in Silica Glass. Journal of Non-Crystalline Solids, 211, 197-202. https://doi.org/10.1016/S0022-3093(96)00647-3

31. Chechetkina, E.A. (1995) Fracture and Fractals in Glasses. Fractal Aspects of Materials, Boston, 28 November-2 December 1994, 397-404.

32. Chechetkina, E.A. (1991) Rawson's Criterion and Intermolecular Interactions in Glass-Forming Melts. Journal of Non-Crystalline Solids, 128, 30-47. https://doi.org/10.1016/0022-3093(91)90774-Z

33. Chechetkina, E.A. (1993) Synergetic Model for the First Sharp Diffraction Peak in Amorphous Substances. Solid State Communications, 87, 171-174.

34. Chechetkina, E.A. (1995) Is There a Relation between Glass-Forming Ability and First Sharp Diffraction Peak? Journal of Physics. Condensed Matter, 7, 3099-3114. https://doi.org/10.1088/0953-8984/7/16/006

35. Chechetkina, E.A. (1996) Key Distinctions in Activation Parameters of Viscous Flow for "Strong" and "Fragile" Glass-Forming Liquids. Journal of Non-Crystalline Solids, 201, 146-149. https://doi.org/10.1016/0022-3093(95)00639-7

36. Chechetkina, E.A. (2016) Self-Organization in Glass: The Synergetic Chemical Bonding Approach. Journal of Optoelectronics and Advanced Materials, 18, 44-49.

37. Messaddeq, S.H., Vallée, R., Soucy, P., Bernier, M., El-Amraoui, M. and Messaddeq, Y. (2012) Self-Organized Periodic Structures on Se-S Based Chalcogenide Glass Induced by Femtosecond Laser Irradiation. Optics Express, 20, 29882-29889. https://doi.org/10.1364/OE.20.029882

38. Nathala, C.S.R., Ajami, A., Ionin, A.A., Kudryashov, S.I., Makarov, S.V., Ganz, T., Assion, A. and Husinsky, W. (2015) Experimental Study of fs-Laser Induced Sub-100-nm Surface Structures on Titanium. Optics Express, 23, 5915-5929. https://doi.org/10.1364/OE.23.005915

39. Höhm, S., Rosenfeld, A., Krüger, J. and Bonse, J. (2015) Laser-Induced Periodic Surface Structures on Titanium upon Single- and Two-Color Femtosecond Double-Pulsed Irradiation. Optics Express, 23, 25959-25971. https://doi.org/10.1364/OE.23.025959

40. Birnbaum, M. (1965) Semiconductor Surface Damage Produced by Ruby Lasers. Journal of Applied Physics, 36, 3688-3695. https://doi.org/10.1063/1.1703071

41. Starbova, K., Dikova, J. and Starbov, N. (1997) Structure Related Properties of Obliquely Deposited Amorphous a- $\mathrm{As}_{2} \mathrm{~S}_{3}$ Thin Films. Journal of Non-Crystalline Solids, 210, 261-266.

https://doi.org/10.1016/S0022-3093(96)00582-0

42. Dembovsky, S.A., Kozyukhin, S.A. and Chechetkina, E.A. (1982) The Experimental Observation and Investigation of Defects in Glass Forming Substances with Selenium as an Example by Viscosimetry in Magnetic and Electric Fields. Materials Research Bulletin, 17, 801-807. https://doi.org/10.1016/0025-5408(82)90031-9

43. Dembovskii, S.A., Chechetkina, E.A. and Kozyukhin, S.A. (1985) Anomalous Effect of Weak Magnetic Fields on Diamagnetic Glassy Semiconductors. JETP Letters, 41, 88-91. 
44. Kolobov, A.V. (2003) Photo-Induced Metastability in Amorphous Semiconductors. Wiley-VCH, Weinheim. https://doi.org/10.1002/9783527602544

45. Hisakuni, H. and Tanaka, K. (1994) Giant Photoexpansion in $\mathrm{As}_{2} \mathrm{~S}_{3}$ Glass. Applied Physics Letters, 65, 2925-2927. https://doi.org/10.1063/1.112533

46. Tanaka, K., Saitoh, A. and Terakado, N. (2006) Giant Photo-Expansion in Chalcogenide Glass. Journal of Optoelectronics and Advanced Materials, 8, 2058-2065.

47. Messadeq, S.H., Li, M.S., Lezal, D. and Ribeiro, S.J.L. (2001) Above Bandgap Induced Photoexpansion and Photobleaching in Ga-Ge-S Based Glasses. Journal of Non-Crystalline Solids, 284, 282-287. https://doi.org/10.1016/S0022-3093(01)00415-X

48. Mendes, A.C., Maia, L.J.Q., Messaddeq, S.H., Messaddeq, Y., Ribeiro, S.J.L. and Li, M.S. (2011) Photoexpansion and Photobleaching Effects in Oxysulfide Thin Films of the $\mathrm{GeS}_{2}+\mathrm{Ga}_{2} \mathrm{O}_{3}$ System. Physica B, 406, 4381-4386. https://doi.org/10.1016/j.physb.2011.08.091

49. Kuzma, V., Bilanych, V., Kozejova, M., Hlozna, D., Feher, A., Rizak, V. and Komanicky, V. (2017) Study of Dependence of Electron Beam Induced Surface Relief Formation on Ge-As-Se Thin Films on the Film Elemental Composition. Journal of Non-Crystalline Solids, 456, 7-11. https://doi.org/10.1016/j.jnoncrysol.2016.10.033

50. Ovshinsky, S.R. (1968) Reversible Electrical Switching Phenomena in Disordered Structures. Physical Review Letters, 21, 1450-1453. https://doi.org/10.1103/PhysRevLett.21.1450

51. Kolomiets, B.T. and Lebedev, E.A. (1963) The Current-Voltage Characteristics of the Point Contact with Glassy Semiconductors. Radiotekhnika i Elektronika, 8, 2097-2098. (In Russian)

52. Gunti, S.R. and Asokan, S. (2010) Thermal and Electrical Switching Studies on $\mathrm{Ge}_{20} \mathrm{Se}_{80-\mathrm{x}} \mathrm{Bi}_{\mathrm{x}}(1 \leq \mathrm{x} \leq 13)$ Ternary Chalcogenide Glassy System. Journal of Non-Crystalline Solids, 356, 1637-1643. https://doi.org/10.1016/j.jnoncrysol.2010.06.028

53. Upadhyay, M. and Murugavel, S. (2013) Correlation between Crystallization Behavior, Electrical Switching and Local Atomic Structure of Se-Te Glasses. Journal of Non-Crystalline Solids, 368, 34-39. https://doi.org/10.1016/j.jnoncrysol.2013.02.028

54. Kumar, R.T.A., Das, S., Lekha, P.C., Asokan, S., Sanjeeviraja, C. and Padiyan, D.P. (2014) Enhancement in Threshold Voltage with Thickness in Memory Switch Fabricated using $\mathrm{GeSe}_{1.5} \mathrm{~S}_{0.5}$ Thin Films. Journal of Alloys and Compounds, 615, 629-635. https://doi.org/10.1016/j.jallcom.2014.07.068

55. Slankamenac, M., Lukic, S.R. and Zivanuv, M. (2010) Electrical and Switching Parameters of Amorphous Chalcogenide Glassy Semiconductors $\mathrm{Cu}_{5}\left(\mathrm{AsSe}_{1.4} \mathrm{I}_{0.2}\right)_{95}$. International Conference on Development and Application Systems, Suceava, 27-29 May 2010, 221-225.

56. Dembovskii, S.A., Vikhrov, S.P., Ampilogov, V.N. and Chechetkina, E.A. (1985) Influence of Weak Magnetic Field on Electrical Switching of Chalcogenide Glassy Semiconductors. Pis' ma Zhurnal Tekhnicheskoi Fiziki, 11, 1267-1271. 